\title{
Toy Models Unifying Dark Energy and Dark Matter
}

\author{
Sandro Silva e Costa* \\ Departamento de Física - UFMT - Av. Fernando Correa da Costa, s/n ${ }^{\circ}-78060-900$ - Cuiabá - MT - Brazil \\ (Received on 12 October, 2005)
}

\begin{abstract}
It is very common to find numerical studies of dark energy and dark matter. Among these, one can find the interesting proposal of unifying dark matter and dark energy with the use of a single component with an 'exotic' equation of state. However, there is a certain shortage of analyses involving exact analytic models following this proposal. Therefore, here are presented examples of simple exact cosmological models which can reproduce some of the desired properties of an unified dark matter/energy fluid.
\end{abstract}

\section{INTRODUCTION}

Today, standard models of the universe are made of five basic components: photons, barions, neutrinos, dark matter and dark energy. The question "what are dark energy and dark matter?" has a standard answer: weakly interacting massive particles (WIMPs) combined either to a cosmological constant $(\Lambda$-CDM models) or to some scalar field (quintessence models).

Another proposal consists of supposing that maybe both dark energy and dark matter could be in fact two different faces of a single component, named quartessence [1]. Basic quartessential cosmological models possess a single fluid whose main characteristic is an exotic equation of state.

Numerical studies of this quartessential scenario can be easily found in the recent literature [2]. But there is a certain shortage of analyses with exact analytical models. Therefore, a basic idea to overcome such deficiency is to choose models where you can do the math first and then think about the physics later.

\section{CLASSICAL SINGLE FLUID MODELS}

Friedmann's equation of General Relativity,

$$
\frac{1}{a^{2}}\left(\frac{d a}{d t}\right)^{2}+\frac{k}{a^{2}}=\frac{8 \pi}{3} \rho+\frac{\Lambda}{3}
$$

plus conservation of energy in an expanding universe,

$$
d\left(\rho a^{3}\right)+p d\left(a^{3}\right)=0
$$

and an equation of state of the kind $p=p(\rho)$ give the behavior of the scale factor $a(t)$.

The simplest models use the linear relation

$$
p=(\gamma-1) \rho,
$$

where $\gamma$ is a free parameter, and this yields the result

$$
\rho=\rho_{0}\left(\frac{a}{a_{0}}\right)^{-3 \gamma},
$$

\footnotetext{
*The author thanks the support of FAPEMAT.
}

from where comes the general solutions

$$
a(\eta)=\left(\frac{8 \pi}{3 k} \rho_{0} a_{0}^{3 \gamma}\right)^{1 / \alpha} \sin ^{2 / \alpha}\left[\frac{\sqrt{k} \alpha}{2} \eta\right]
$$

with $\alpha \equiv 3 \gamma-2$ and $\gamma \neq 2 / 3$, and

$$
a(\eta)=\exp \left[\left(\frac{8 \pi}{3} \rho_{0} a_{0}^{2}-k\right)^{1 / 2} \eta\right]
$$

for $\gamma=2 / 3$, both valid for $\Lambda=0$ and where $\eta$ is a "conformal time'.

If $\Lambda>0$ it is possible to obtain analytical solutions only for some values of $\gamma$. For example,

$$
a=\left[\sqrt{\frac{8 \pi}{3} \rho_{0} a_{0}^{4}}\left(\frac{\sinh 2 t \sqrt{\frac{\Lambda}{3}}}{\sqrt{\frac{\Lambda}{3}}}\right)-k\left(\frac{\sinh t \sqrt{\frac{\Lambda}{3}}}{\sqrt{\frac{\Lambda}{3}}}\right)^{2}\right]^{1 / 2}
$$

for $\gamma=4 / 3$, and

$$
a=\sqrt{\frac{8 \pi}{3} \rho_{0} a_{0}^{2}-k}\left(\frac{\sinh t \sqrt{\frac{\Lambda}{3}}}{\sqrt{\frac{\Lambda}{3}}}\right)
$$

for $\gamma=2 / 3$.

A $0^{\text {th }}$ order cosmological test for quartessential models is to compare them to such 'classical' single fluid cosmological solutions.

\section{EXOTIC EQUATIONS OF STATE}

The most usual exotic equation of state found in the literature is a generalization of the Chaplygin gas,

$$
p=-M \rho^{-\alpha},
$$

with $M$ and $\alpha$ parameters to be determined numerically and/or statistically. But why use such equation? From energy conservation comes that

$$
\rho=\rho_{0}\left[\left(1-\frac{M}{\rho^{1+\alpha}}\right)\left(\frac{a}{a_{0}}\right)^{-6}+\frac{M}{\rho^{1+\alpha}}\right]^{1 /(1+\alpha)},
$$




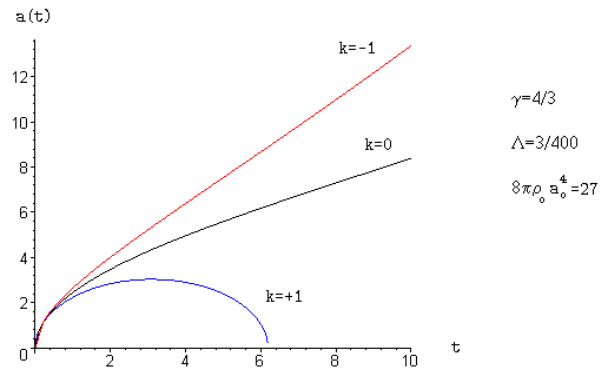

FIG. 1: Graphs of $a(t)$ for a classical single fluid model with $p=\rho / 3$ and $\Lambda \neq 0$.

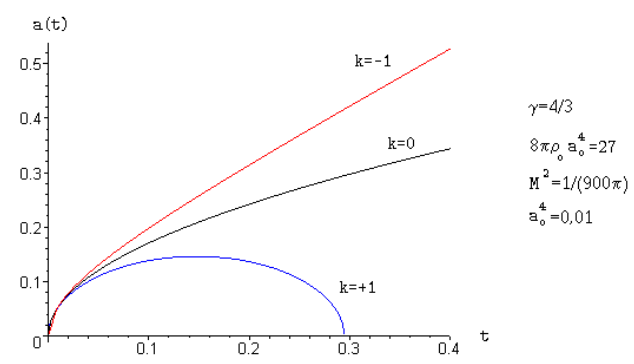

FIG. 2: Graphs of $a(t)$ for a model with $p=\rho / 3-M \rho^{1 / 2}$ and $\Lambda=0$.

and for $\alpha=1$ one has

$$
a \rightarrow \infty: \rho \rightarrow M, a \rightarrow 0: \rho \rightarrow a^{-3} .
$$

This kind of behavior can be implemented with another approach: by adding non-linear terms to the usual linear equation of state, i.e.,

$$
p=(\gamma-1) \rho+f(\rho) .
$$

The non-linear term $f(\rho)$ can be chosen to produce analytical results and mimic both dark matter and energy.

Therefore, the steps to be followed are:

1. firstly select exotic equations of state which give analytical solutions;

2. then see which solutions can mimic dark matter and energy, passing through cosmological tests of $0^{\text {th }}, 1^{s t}, 2^{\text {nd }}$ and higher orders.
One example of a generalized equation of state is

$$
p=(\gamma-1) \rho-M \rho^{-\alpha},
$$

with $\alpha \neq-1$. From this, one obtains, by energy conservation, with $\gamma \neq 0$,

$$
\rho=\rho_{0}\left[\left(1-\frac{M^{\prime}}{\gamma}\right)\left(\frac{a_{0}}{a}\right)^{3 \gamma(1+\alpha)}+\frac{M^{\prime}}{\gamma}\right]^{\frac{1}{1+\alpha}},
$$

where $M^{\prime} \equiv M \rho_{0}^{-(1+\alpha)}$. Choosing, for example, $\alpha=-1 / 2$ and $\gamma=\frac{4}{3}$ one has the solution

$$
\begin{aligned}
a= & {\left[a_{0}^{2}\left(\frac{4}{3 M^{\prime}}-1\right)\left(e^{\sqrt{6 \pi \rho_{0}} M^{\prime} t}-1\right)\right.} \\
& \left.-k\left(\frac{\sinh \sqrt{\frac{3 \pi}{2} \rho_{0}} M^{\prime} t}{\sqrt{\frac{3 \pi}{2} \rho_{0}} M^{\prime}}\right)^{2}\right]^{\frac{1}{2}},
\end{aligned}
$$

while for $\gamma=\frac{2}{3}$ one has

$$
\begin{aligned}
a= & 2 \pi a_{0} \rho_{0} M^{\prime}\left(1-\frac{3 M^{\prime}}{2}\right)\left(\frac{\sinh \sqrt{\frac{3 \pi \rho_{0}}{2}} M^{\prime} t}{\sqrt{\frac{3 \pi \rho_{0}}{2}} M^{\prime}}\right)^{2} \\
& +\left[\frac{8 \pi a_{0}^{2} \rho_{0}}{3}\left(1-\frac{3 M^{\prime}}{2}\right)^{2}-k\right]^{1 / 2} \frac{\sinh \sqrt{6 \pi \rho_{0}} M^{\prime} t}{\sqrt{6 \pi \rho_{0}} M^{\prime}} .
\end{aligned}
$$

These results are comparable to the ones obtained in classical single fluid models (see Figures 1 and 2) and in models with $\Lambda=\Lambda(t)$ [3].

The search for functions $f(\rho)$ which produce analytical results can go on and on. But for all the proposals of exotic equations of state one must check what kind of energy/matter these equations can represent, their stability, growth of perturbations, etc [4].

\section{CONCLUSIONS}

Since the hunting season for dark matter and dark energy models is still open, several proposals must be considered. Quartessence is a simple idea and as such can be used as food for thoughts (even if wrong). But does quartessence have a future? The answer may be on the next corner, and analytical results may help, providing forward steps.
[1] M. Makler, S.Q. Oliveira, and I. Waga. Phys. Lett. B 555, 1 (2003).

[2] R. R. R. Reis, M. Makler, and I. Waga. Class. Quant. Grav. 22, 353 (2005).
[3] H. A. Borges and S. Carneiro. gr-qc/0503037 (2005).

[4] S. S. Costa. Article in preparation. 